\title{
Le Baiser de la Belle au bois dormant ou : des péripéties encourues par la géographie linguistique depuis Jules Gilliéron
}

Hans Goebl

\section{(2) OpenEdition \\ Journals}

Electronic version

URL: http://journals.openedition.org/corpus/2311

DOI: 10.4000/corpus.2311

ISSN: $1765-3126$

\section{Publisher}

Bases ; corpus et langage - UMR 6039

\section{Printed version}

Date of publication: 1 January 2013

Number of pages: 61-84

ISSN: 1638-9808

\section{Electronic reference}

Hans Goebl, «Le Baiser de la Belle au bois dormant ou : des péripéties encourues par la géographie linguistique depuis Jules Gilliéron », Corpus [Online], 12 | 2013, Online since 13 May 2014, connection on 08 September 2020. URL : http://journals.openedition.org/corpus/2311 ; DOI : https://doi.org/ $10.4000 /$ corpus. 2311 


\section{Le Baiser de la Belle au bois dormant ou : des péripéties encourues par la géographie linguistique depuis Jules Gilliéron ${ }^{1}$}

Hans GOEBL

Universität Salzburg

\section{Remarque préliminaire}

Le but de cet article est d'analyser de plus près le substrat méthodique et aussi idéologique sur lequel reposent l'ALF et la pensée géolinguistique de son créateur Jules Gilliéron (18541926). J'ajoute, en guise de préambule, que l'ALF remplit, de par sa structure bidimensionnelle très claire $\left(638^{2}\right.$ points d'enquête fois 1421 cartes d'atlas de la série $\mathrm{A}^{3}$ ) tous les prérequis d'une base de données classique et aussi - à cause de sa finalité linguistique bien définie - ceux d'un corpus (dialectal), dont il est couramment question dans ce volume. A la lumière de la rigueur des choix méthodiques sur lesquels repose la genèse de l'ALF, l'auteur estime que l'histoire post-gilliéronienne de la géographie linguistique est marquée par des tendances qui, en dernière analyse, sont allées à l'encontre des intentions générales de Gilliéron et du plan méthodique de l'ALF.

1 Révison stylistique de mon texte français : Lily Ditz-Fuhrich, Université de Salzbourg. Qu'elle reçoive ici l'expression de ma parfaite reconnaissance.

2 Le nombre exact des points d'enquête de l'ALF est 638. Comme au pointALF 284, Saint-Pol-sur-Ternoise, Edmond Edmont a fait deux enquêtes, le nombre des enquêtes faites pour l'ALF s'élève à 639. La confusion très fréquente entre ces deux chiffres est due à des imprécisions contenues déjà dans la fameuse " Notice servant à l'intelligence des cartes », publiée par J. Gilliéron en 1902, donc au moment de la parution du premier fascicule de l'ALF.

3 Pour les trois séries de cartes de l'ALF (A-C) voir les tableaux respectifs dans Pop/Pop 1959, 96-118.

Corpus $\mathrm{n}^{\circ} 12$ « Dialectologie : corpus, atlas, analyses » (2013), 
H. GOEBL

\section{En vainquant le choléra par voie cartographique : l'exploit du docteur John Snow en 1854}

Nous sommes au début du mois de septembre de 1854; la scène a lieu à Londres où le choléra sévit dans les quartiers populaires, en particulier dans celui de Soho. Quant à l'origine et aux modes de diffusion de cette maladie épidémique, les avis des médecins de l'époque sont partagés. Les uns favorisent la thèse selon laquelle la contamination par le choléra dépend du contact des humains avec les mauvaises odeurs (appelées alors miasmes) circulant dans l'atmosphère, alors que les autres pensent que c'est la consommation d'eau infectée qui provoque l'apparition de la maladie en question.

Le district de Soho, un des quartiers londoniens les plus touchés par le choléra, est placé sous la responsabilité du docteur John Snow (1813-1858). C'est un esprit éclairé qui, quant à la diffusion du choléra défend l'idée de la contamination par l'eau insalubre. Devant la nécessité d'enrayer d'urgence la propagation du choléra dans un triangle situé entre Broad Street, Golden Square and Work House, il décide de cartographier avec précision les trois facteurs suivants :

- le réseau des ruelles du quartier,

- les emplacements des pompes à eau utilisées avec régularité et

- le nombre et les endroits des décès survenus quotidiennement.

Pour ce faire, il organise un réseau d'informants et de collaborateurs tout en les sommant à lui fournir les informations nécessaires jour après jour et à une heure établie à l'avance. La mise en carte des données recueillies sera effectuée par luimême.

La préparation et le résultat de cette opération ont reçu une notoriété universelle. On en trouve des mentions dans tous les manuels de cartographie thématique ${ }^{4}$ et aussi dans beaucoup d'ouvrages épistémologiques.

En effet, le docteur Snow a réussi, moyennant cette mise en carte méticuleuse, à repérer une pompe à eau située au

4 Cf. Robinson 1982, 176-180, Palsky 1996, 81-84 et Tufte 1997, 27-37. 
coin entre la Broad Street et la Cambridge Street autour de laquelle le nombre des décès dus au choléra culminait. Sans tarder, il en fit enlever la manivelle, après quoi le nombre des morts du choléra diminua rapidement.

La démarche et l'expérience du docteur John Snow sont exemplaires à plus d'un égard :

- du point de vue théorique : il avait une théorie precise qui lui servait de fil conducteur intellectuel : c'était, d'un côté, la contagion de l'eau et, de l'autre, la conviction que la diffusion de la maladie dût obéir aux impératifs de l'espace.

- du point de vue méthodique : étant au courant, de loin ou de près, de la cartographie thématique de son temps, il savait que l'utilisation réfléchie de cartes pouvait résoudre des problèmes qui autrement s'avéraient comme insolubles.

- du point de vue opérationnel : il savait comment organiser la collecte des données dont il avait besoin.

Les actions de Snow étaient dictées par une finalité très claire - ciblée sur la découverte du secret de la propagation du choléra - et par les nécessités pratiques de la récolte de données empiriques et de leur mise en carte successive.

Dans l'optique de l'historien qui, en ce moment, est la nôtre, il appert donc que la victoire sur le choléra à Londres en 1854 dépendait de trois facteurs :

- de la théorie épidémique correcte du docteur Snow,

- de ses connaissance cartographiques et

- de son habilité opérationnelle.

Evidemment, ce sont trois prérequis dont dépend également le bon maniement de n'importe quel corpus (géo)linguistique digne de ce nom.

Le lecteur attentif comprendra aisément que la Belle au bois dormant que le docteur Snow a réussi à trouver, réveiller et embrasser, est représentée par le noyau correct de sa théorie relative à la propagation du choléra. Nous verrons par la suite que le monde des dialectes offre de semblables chances aux linguistes animés de penchants théorisants similaires. 
H. GOEBL

\section{Un regard critique sur la proto-histoire de l'ALF}

Alors que pour l'AIS de Karl Jaberg (1877-1958) et Jakob Jud (1882-1952) et aussi pour beaucoup d'autres atlas linguistiques nationaux (comme l'atlas roumain ALR ou l'atlas ibérique ALPI) ou régionaux publiés après l'ALF, nos connaissances sur leurs conceptions et réalisations sont relativement bonnes, les informations dont nous disposons sur la proto-histoire, la collecte des données et l'impression de l'ALF sont plutôt limitées. Elles dépendent entièrement de ce que Jules Gilliéron a confié aux pages introductives de la fameuse "Notice servant à l'intelligence des cartes » (de 1902), de quelques brèves observations disséminées dans ses nombreuses publications géolinguistiques basées sur les données de l'ALF ${ }^{5}$ et des observations supplémentaires, pieusement réunies et publiées par Sever Pop (19011961) et sa fille Rodica Doina, longtemps après la mort du maître, survenue en 1926.

Il s'y ajoute les travaux historiques de la regrettée Marie-Rose Simoni-Aurembou, basés, entre autres, sur l'introspection du legs Gilliéron déposé à la Bibliothèque Nationale à Paris ${ }^{6}$, et aussi les apports de l' "Ecole de Louvain », placée sous la direction de Pierre Swiggers ${ }^{7}$.

En 2005, ce bilan a été complété par l'ouvrage, richement illustré, de Jean Le Dû, Yves Le Berre et Guylaine BrunTrigaud, qui, en dernière analyse, repose sur l'usage fait des cartes de l'ALF par un grand admirateur non romaniste de ce chef d'oeuvre de la romanistique, à savoir par le chanoine François Falc'hun (1909-1991), jadis professeur de Philologie Celtique aux Universités de Rennes (1951-1967) et de Brest (1967-1981).

C'est surtout de la somme dialectologique bien connue de Sever Pop, publiée en deux volumes en 1950, qu'il ressort avec netteté que, durant toute la durée du XIX ${ }^{\mathrm{e}}$ siècle, les intérêts dialectologiques ne cessaient de se concrétiser, certes, dans les

5 Pour la bibliographie complète et une synopse thématique des travaux de Gilliéron cf. Pop/Pop 1959, 128-146 et 20-33.

6 B.N. de Paris : cotes du legs de Gilliéron : NAF 11971-12030.

7 Cf. Lauwers/Simoni-Aurembou/Swiggers 2002, passim. 
majeures cultures d'Europe, mais que c'était surtout la France où cet essor d'idées était " général » puisqu'il était intimement lié à l'éclosion d'une conception particulière de l'espace national.

Nous entendons par là une riche gamme de préoccupations empiriques et analytiques déployées autour des territoires du Roi (ou de l'Empereur) de France d'abord et de la République française par la suite, de la part d'économistes, géographes, cartographes, géodètes, militaires et ingénieurs de toute sorte, visant toutes à maîtriser les multiples avatars de l'espace de la France par l'accumulation et le raffinement du savoir relatif à ses propriétés, fonctions, richesses et aussi à ses limitations et contraintes ${ }^{8}$.

On connaît l'intérêt du XVIII ${ }^{\mathrm{e}}$ siècle et aussi de l'administration napoléonienne (et post-napoléonienne) pour la cueillette (systématique) de traductions de la Parabole de l'Enfant Prodigue $^{9}$ qui servaient à déceler la bigarrure linguistique (mieux : dialectale) de la France et des pays récemment conquis par les armes. Rappelons, à ce propos, les noms de CharlesEtienne Coquebert de Montbret (1755-1831) et de son fils Eugène-Barthélémy (1785-1849) à qui l'on doit la première collecte systématique et standardisée de données dialectales pour des espaces tant très étendus que bien circonscrits. Malheureusement, le résultat de la mise en carte de ces recherches dont Coquebert le Jeune parle explicitement en $1831^{10}$, n'a pas été conservé.

Un autre fait doit être évoqué avec insistance. C'est la départementalisation de la France, survenue très peu de temps après l'éclatement de la Révolution française ${ }^{11}$. La mise en place

\footnotetext{
8 Voir l'historique que nous en avons fait en 2006 (passim).

9 Cf. Simoni-Aurembou 1989, passim.

10 «C'était au moyen de ces renseignemens recueillis avec soin et notés avec scrupule que l'on a marqué sur des cartes particulières des départemens, et par suite sur une carte générale de la France, les limites géographiques des différens idiomes, [notation en caractères gras : H. G.] et qu'on avait donné dans l'Annuaire des Longitudes le tableau qui y a figuré en 1809 et années suivantes, tableau qu'il ne sera pas inutile peutêtre de reproduire ici. » (Coquebert de Montbret/Labouderie 1831, 14-15).

11 Cf. Ozouf-Marignier 1989 et Roncayolo 1992.
} 


\section{H. GOEBL}

- réalisée avec beaucoup d'aplomb administratif et politique du nouveau réseau départemental en substitution de l'ancienne bigarrure enchevêtrée des provinces, comtés et duchés de type féodal, a créé non seulement une nouvelle conscience de l'espace national basée sur l'idée du quadrillage, de l'équidistance et de la parité fondamentale des nouvelles subdivisions territoriales, mais a également fourni de nouvelles possibilités empiriques pour l'administration nationale et tous les intellectuels oeuvrant à son service.

En effet, la liste des 75 premiers départements représente, en dernière analyse, un échantillon standardisé de premier ordre de l'espace total de la France auquel s'est superposé plus tard un échantillon supplémentaire comprenant un nombre - d'ailleurs fort limité - d'agglomérations majeures de chaque département. Or, il est peu connu parmi les linguistes que durant tout le XIX ${ }^{\mathrm{e}}$ siècle il s'est formé en France une cartographie officielle très développée qui, d'un côté, reposait sur d'intenses recherches empiriques thématiquement très diversifiées, réalisées sur la base du réseau départemental élargi par les lieux centraux de chaque département, et, de l'autre, sur une visualisation de plus en plus élaborée et efficace des données recueillies. En 1996, le géographe français Gilles Palsky en a fait le point dans une synthèse richement documentée et illustrée.

De toutes ces activités géographiques et cartographiques il est né une sensibilisation universelle pour l'espace de la France dont on trouve bien sûr des retombées aussi sur le plan de la dialectologie. Mentionnons à ce propos les débats relatifs au parcours précis de la frontière entre les domaines d'Oïl et d'Oc dans le cadre duquel s'inscrivent les recherches empiriques sur le terrain qui - ayant le rang d'une mission officielle subventionnée par le Ministère de l'Intérieur même - ont été effectuées par Charles de Tourtoulon (1836-1913) et Octavien Bringuier (1829-1875) entre 1873 et 1875 , suivies en 1876 - c'est-à-dire déjà après la mort d'O. Bringuier - de la publication d'un rapport et d'une carte qui n'englobait malheureusement que la partie occidentale de l'espace parcouru ${ }^{12}$.

12 Voir à ce sujet notre contribution de 2004 (passim) et Storost 2008 (124152). 
Signalons en outre que la bipartition linguistique de la France en Oc et Ö̈l, bien connue depuis longtemps, a fini par devenir une pomme de discorde scientifique d'un côté et un « risque » pour l'unité de la Nation de l'autre, surtout en temps de crise. Ce conflit, surgi entre les philologues Paul Meyer (1840-1917) et Gaston Paris (1839-1903) d'un côté, et le dialectologue italien Graziadio Isaia Ascoli (1829-1907) de l'autre. portait sur la question de savoir si l'articulation dialectale de la France comportait seulement deux compartiments (= Oc + Ö̈l) comme le voulaient $\mathrm{P}$. Meyer et G. Paris, ou même trois (= Oc + Oil + franco-provenzale), comme le prétendait G. I. Ascoli ${ }^{13}$ à l'appui de preuves dont le bien-fondé est de nos jours universellement reconnu ${ }^{14}$.

Pour la date de l'éclatement de ce conflit (cf. Meyer 1875) le contexte historico-politique n'est pas sans importance : elle se situe quatre ans après la fin de la guerre franco-prussienne de 1870/71, donc dans une période riche en soubresauts nationalistes de toute sorte, et où l'idée de cohésion et de l'indivisibilité du sol national avait le rang d'une obsession omniprésente.

\section{Un coup d'œil rétrospectif dans l'atelier de Jules Gilliéron}

Jules Gilliéron est né en 1854 dans la Suisse romande (à La Neuveville, aux bords du Lac de Bienne), fut élevé dans sa patrie et s'est installé définitivement à Paris en 1876, donc à l'âge de 22 ans. En 1886, à 32 ans, il a sollicité et reçu la citoyenneté française en tant que Maître de conférences à l'Ecole Pratique des Hautes Etudes, charge qu'il occupa à partir de 1882 jusqu'en 1926, l'année de sa mort. En outre, il enseigna comme professeur d'allemand au Lycée Chaptal entre 1878 et 1897.

Grâce à ces deux fonctions, il était en contact étroit et permanent avec les élites intellectuelles de la France et pouvait donc se familiariser avec tous les acquis intellectuels, culturels et administratifs de la France impériale, royale et républicaine $\mathrm{du}$ XIX ${ }^{\mathrm{e}}$ siècle. Quant au penchant indéniable de Gilliéron pour la géographie, il ne faut non plus oublier que son père Jean

13 Cf. Ascoli 1874.

14 Pour une présentation critique de ces discussions cf. Goebl 2010, passim. 


\section{H. GOEBL}

Victor (1826-1890) était géologue ${ }^{15}$. Il est donc tout à fait probable que la rigueur géo- et cartographique de l'ALF dépend, chez Gilliéron, à parts égales, de l'héritage spirituel de son père et de ce dont il a pu prendre acte à Paris à partir de 1876 .

Il est bien connu que Gilliéron, fort de son expérience juvénile avec le «Petit atlas phonétique du Valais roman » (publié en 1881) a mûri l'idée de l'ALF dans un perpétuel échange d'idées avec le célèbre philologue G. Paris qui a fini par lui en fournir les subsides nécessaires voire indispensables, tant pour la réalisation des enquêtes que pour l'impression effectuée à Mâcon par les soins de l'imprimerie Protat Frères ${ }^{16}$.

Quant à la maturation des idées de Gilliéron relatives à l'ALF, il faut admettre, chez lui, la genèse de deux « convictions » qui, dans la rétrospective, peuvent également être qualifiées de théories:

1) que l'espace de la France - mieux : de la Galloromania - est une espèce de "machine» régie, entre autres, par les mécanismes du langage dialectal. Toutes les expériences antérieures, faites au cours du XIX ${ }^{\mathrm{e}}$ siècle par les géographes, économistes, démographes et «statistiques » ont démontré que tel était le cas pour beaucoup de variables d'origine humaine, économique ou technique.

2) que les locuteurs dialectophones - bilingues (dialecte local et français) déjà à des pourcentages très élevés à la fin du $\mathrm{XIX}^{\mathrm{e}}$ siècle - sont capables d'extraire, au moment de l'enquête, de leur compétence multiple la composante dialectale, et de la présenter à l'enquêteur sous une forme qui, dans une optique moderne, peut être qualifiée comme étant "métrologiquement décantée ». C'est justement ce processus de "décantation» pragmalinguistique qui se déroule pendant l'acte de traduction, faite par le sujet interviewé, auquel on doit attribuer le haut degré d'intercomparabilité des données répertoriées sur les 1421 planches de l'ALF.

15 Cf. Pop/Pop 1959, 5.

16 L'impression de l'ALF a été réalisée sous la surveillance particulière de Georges Protat (1857-1923) et constitue, en tant que telle, un véritable chef d'œuvre de la typographie ; cf. Lequin 1924, 53 et Gilliéron 1902, $9-10$. 
N'oublions pas que la plus sévère des consignes qu'avait reçues E. Edmont de J. Gilliéron pour la réalisation des enquêtes, était de toujours enregistrer la première réponse et d'éviter toute extorsion des répliques.

C'est à cette sobriété discursive qu'est due, entre autres, la rapidité des enquêtes faites pour l'ALF. En effet, E. Edmont a employé, tout compte fait, seulement 1351 jours pour réaliser les 639 enquêtes de l'ALF $^{17}$. Ceci donne la moyenne de 2,11 jours pour chaque enquête, dont la longueur variait entre 1421 (pour la série A) et 1920 (pour la série C) questions. Etant donné le haut degré de fiabilité des réponses ainsi recueillies - et qui avait été démontré très souvent - l'expéditivité inégalable d'Edmont a depuis toujours suscité ma plus haute admiration.

A partir de 1902, les dialectologues - tant en France qu'à l'étranger - pouvaient donc se servir d'un corpus qui présentait les atouts suivants :

1) relevé hautement standardisé des réponses auprès de sujets interviewés à l'aide d'un questionnaire soigneusement préparée et préalablement testée, par les soins d'une personne dotée d'une fiabilité personnelle et d'une ténacité physique extraordinaires,

2) intercomparabilité parfaite des données transcrites,

3) données très complètes et, de ce fait, peu lacunaires.

Tout compte fait, je n'hésite pas à qualifier l'entreprise de l'ALF comme une sorte de géodésie faite avec des moyens linguistiques, et de l'appeler ainsi glotto-géodésie ${ }^{18}$.

J. Gilliéron et son imprimeur Georges Protat ont en outre ouvert la voie à un dépouillement efficace des planches de l'ALF par la mise à disposition de cartes muettes ${ }^{19}$ en format

$17 \mathrm{Cf}$. Brun-Trigaud/Le Berre/Le Dû 2005, 21 où se trouvent les dates des huit missions exploratoires qu'Edmont avait accomplies entre le $1^{\text {er }}$ août 1897 et le 10 août 1901.

18 Rappelons que le mot savant géodésie, d'origine grecque, signifie « subdivision du territoire »: < gr. gés « terre » et dáinymi « partager, diviser ».

19 La valeur heuristique des cartes muettes est inestimable. Ceci appert, entre autres, par un coup d'œil comparatif à d'autres philologies modernes qui, comme p. ex. les études germaniques et anglaises, ne connaissent pas cet instrument. Mais aussi au sein des études romanes les seules entreprises d'atlas à mettre à disposition des cartes muettes étaient 


\section{H. GOEBL}

A3 dont l'utilité pratique a tout de suite été reconnue par les spécialistes. C'est ainsi que le romaniste suisse Karl Jaberg en a fait une description détaillée déjà en 1906 dans la «Zeitschrift für romanische Philologie ».

N'oublions pas non plus que l'entier « système ALF » comprenait, surtout après la publication des premiers fascicules à partir de 1902, aussi l'enseignement «magnétique » de Jules Gilliéron à l'Ecole Pratique des Hautes Etudes auquel participait la fine fleur des romanistes et linguistes européens de l'époque ${ }^{20}$.

Le propre de cet enseignement et aussi de la productivité scientifique de J. Gilliéron lui-même était la prise en compte « enveloppante » des données de l'ALF qui, de ce fait, ont toujours été perçues non seulement comme « systémiques » en tant que telles, mais aussi comme reflets d'un «système » extérieur, que ceci soit réel, sous-jacent ou même caché. Rares étaient ceux qui se bornaient à voir, dans les données de l'ALF, un amas de données dialectales hétéroclites dont le seul avantage était leur datation et localisation excellentes.

\section{La fortune ultérieure de l'ALF}

L'usage qui a été fait des données publiées de l'ALF s'est développé dans deux directions: dans celle d'un dépouillement intégral de planches isolées ou - très rarement - combinées de l'ALF, et dans celle de l'utilisation particulière de ses données telle qu'elle était, entre autres, la pratique courante dans l'établissement du $\mathrm{FEW}^{21}$.

Il est curieux de voir que Jules Gilliéron lui-même - qui pourtant venait de rassembler un corpus ultra-systémique - se limitait, dans ses propres travaux géolinguistiques, à des analyses

l'ALF, l'AIS, l'ALR et - l'ALD (bien sûr). Pour toutes les autres, les utilisateurs devaient s'en procurer « avec les moyens du bord ».

20 Voir la liste y ayant trait dans Pop/Pop 1959, 53-63.

21 Ceci n'a nullement perturbé la bonne qualité de la coexistence du filon « néogrammairien » et de la géographie linguistique de J. Gilliéron dans la rédaction des articles du FEW. Une preuve tangible de ce fait est la double dédicace du premier volume du FEW à Wilhelm Meyer-Lübke (1861-1936) et à Jules Gilliéron par Walther von Wartburg (1888-1971), l'auteur du FEW. 
psychologisantes de cartes-ALF isolées alors que des chercheurs germanophones - à commencer par Karl Jaberg ${ }^{22}$ - ont très tôt cédé à la tentation globalisante pour dresser des synthèses isoglottiques et des cartes à densité dont on ne trouve aucune

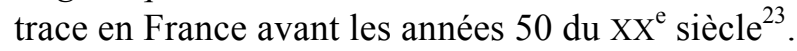

Les reproches n'ont pas tardé à pleuvoir sur l'ALF. Mais ces reproches ne visaient pas son caractère systémique comme tel, mais une prétendue défectuosité des transcriptions, des lacunes dans le questionnaire et surtout le maillage trop lâche de son réseau. C'étaient des voix qui culminaient dans la revendication d'une plus grande "authenticité » documentaire des données de l'atlas ${ }^{24}$.

On connaît les résultats de ces tentatives : c'est la splendide série des "Nouveaux Atlas Linguistiques de la France » $(\mathrm{NALFs})^{25}$ qui tous ont été faits pour étudier « de plus près » les patrimoines dialectaux de provinces et régions historiquement typées, tant par le resserrement du réseau d'exploration, la « popularisation » des méthodes d'enquête (moyennant l'usage du patois local, la conversation dirigée ou même libre à la place de l'application d'un questionnaire standardisé) que par l'adaptation du contenu du questionnaire respectif aux exigences (factuelles, linguistiques, ethnographiques etc.) de la région concernée.

Malheureusement l'écrasante majorité des quelque 30 NALFs ainsi établis a été réalisée dans l'oubli presque complet des consignes données au départ par Albert Dauzat, l'instigateur de l'idée des NALFs. Comme les questionnaires des différents NALFs ne disposent même pas de 10 questions communes ${ }^{26}$, il est complètement impossible de s'en servir pour vérifier, à l'aide d'un réseau plus serré, la persistance, à la distance de quelque 50 ans, des structures dialectales globales et, partant,

22 Cf. l'excellente présentation de l'ALF rédigée par K. Jaberg dès 1908.

23 Voir à ce sujet aussi les ouvrages séminaux de Karl von Ettmayer (1924) et Karl Jaberg (1936). Pour un historique détaillé de cette évolution hétérogène cf. Goebl 2004, passim.

24 Cf. à cela Dauzat 1949, 5-30.

25 Sous les auspices du CNRS français, cette série fut rebaptisée, à partir de 1980, en « Atlas linguistiques de la France par régions ».

26 Cf. Billy 1993, passim. 


\section{H. GOEBL}

sous-jacentes de la Galloromania dont l'existence a été révélée par l'ALF et prouvée dans les détails par les apports de la dialectométrie ${ }^{27}$.

J'ajoute, entre parenthèses, que la dialectométrie considère les structures de profondeur citées ci-dessus comme émanation directe d'une activité langagière particulière des locuteurs visant à s'approprier linguistiquement l'espace dans lequel ils vivent. Pour la saisie terminologique de cet effet, j'utilise, depuis un certain temps, le concept de la "gestion basilectale de l'espace par l'homo loquens $»^{28}$.

L'on sait que K. Jaberg et J. Jud ont remplacé, dans la rédaction du questionnaire de leur atlas italo-suisse AIS, la sobriété sémantique du questionnaire de l'ALF par une allure beaucoup plus ethnographique, tout en répondant, de cette manière, au filon de recherche "Wörter und Sachen » de trempe onomasiologique $^{29}$. Ce faisant, Jaberg et Jud - qui n'ont jamais cessé de se considérer comme élèves de J. Gilliéron - n'ont d'ailleurs pas forcé la dose. Ce qui fait que les planches de l'AIS révèlent, comme celles de l'ALF, l'existence de structures dialectales de profondeur très nettes ${ }^{30}$.

\section{De l'atlas basilectal à l'atlas de répertoire}

Rappelons d'entrée de jeu que malgré la frénésie documentaire (ou faudrait-il dire : documentaliste ?) déployée dans la période des NALFs, la volonté de déceler, à côté de la documentation " réelle", "positive» et audible, aussi des structures systémiques de profondeur ne s'est pas éteinte. C'est justement dans le cadre de l'atlas linguistique gascon (ALG) et sous l'impulsion d'un fourmillement inouï de variantes dialectales y ayant trait, qu'est née, dans la tête de son auteur Jean Séguy (1914-

27 Voir à ce sujet surtout mes travaux dialectométriques relatifs à l'ALF de 1984, 2002 et 2003.

28 J'évoque ce concept dans toutes mes publications dialectométriques depuis 2000 : cf. la liste bibliographique sous : https ://www.sbg.ac.at/rom/ people/prof/goebl/dm_publi.htm

29 Je renvoie, à ce sujet, à l'excellent volume introductif de l'AIS (Jaberg/Jud 1928).

30 Cf. Goebl 1984 et 2008. 
1973) l'idée de synthétiser cet apparent chaos et de le mettre en formule. Xavier Ravier, son compagnon de travail de longue date, a relaté à ce sujet les propos suivants de Jean Séguy: "Désormais je peux crever tranquille. L'idée fixe qui me hantait depuis trente ans est réalisée; à partir de 47 millions de chiures de mouche scrupuleusement intégrées, arriver, par une série d'abstractions à la fois mathématiques et réalistes, à faire tenir le gascon dans une formule ou un schéma. » (Ravier 1976, 390).

Depuis 1971 ou $1973^{31}$, la dialectométrie a fait d'énormes progrès, et ceci aussi en dehors de la romanistique. Mais c'est également la dialectométrie - mieux : l'essai d'appliquer les méthodes dialectométriques à proprement parler - qui a révélé certaines faiblesses de recueils de données plus récents qui, à l'instar de l'ALF ou de l'AIS, se décorent du qualificatif d' " atlas » ou « atlante » (etc.). Beaucoup de ces entreprises ont abandonné les principes de la collecte sélective de données en se proposant de ne pas se limiter à des réponses jugées dialectales par les locuteurs eux-mêmes, mais de s'adonner allègrement à la documentation de toute l'ampleur des compétences - forcément multiples - des sujets. C'est de là qu'est née l'idée de l' « atlante repertorio », cultivée surtout en Italie. Je cite à ce propos les entreprises du « Nuovo Atlante del Dialetto e dell'Italiano per Regioni » (NADIR) conçu par Alberto Sobrero, de l' «Atlante linguistico siciliano » (ALS) ${ }^{32}$, promu par Giovanni Ruffino, et de l' "Atlante linguistico della Campania » $(\mathrm{ALCam})^{33}$, dirigé par Edgar Radtke.

Une des conséquences pratiques de l'élargissement programmatique du rayon d'action de la collecte des données était la multiplication - souvent de forme carrément incontrôlable de variantes (réponses multiples). Ce qui a échappé complètement aux promoteurs de cette nouvelle stratégie était le fait qu'avec l'élargissement de la vertu documentaire des enquêtes allait de pair la diminution de l'intercomparabilité des données

31 Les deux articles séminaux de Séguy datent de 1971 et 1973.

32 Voir le site web de l'ALS : http://www.csfls.it/?id=34.

33 Voir le site web de l'ALCam : http://www.alcam.de/. 


\section{H. GOEBL}

recueillies et, partant, de leur caractère systémique, et qu'ils emboîtaient, de ce fait, le chemin dictionnairique que Gilliéron qualifiait avec mépris de la façon suivante : «dictionnaire cimetière $»$.

«Qui trop embrasse, peu étreint»: il y a des vérités scientifiques aussi dans les proverbes les plus banals.

Evidemment, une documentation faite sous le signe de l' «atlante repertorio" est plus authentique ou naturaliste ${ }^{34}$ qu'une enquête réalisée sur le modèle de l'ALF. Je ne nie aucunement l'utilité de l'authenticité et du naturalisme pour certains propos. Mais j'insiste sur le fait que ces propos ne sauront jamais répondre aux exigences théoriques d'une base de données compilée à la gilliéronienne.

Depuis quelque temps je suggère aux tenants de l' " atlante repertorio » de ne plus utiliser le qualificatif d'atlas (ou atlante) pour leurs recueils afin d'éviter des confusions malencontreuses $^{35}$.

Le même scepticisme concerne l'outil empirique des ethnotextes dont il existe, dans différents pays européens, des collections très riches qui permettent de couvrir l'étendue entière des pays en question. Certes, la valeur documentaire des ethnotextes est hors de doute ; mais ils obéissent à des impératifs empiriques très différents de ceux d'une enquête standardisée à la façon de l'ALF si bien qu'il est complètement vain de vouloir créer, à partir d'un grand nombre d'ethnotextes éparpillés sur le terrain, l'équivalent fonctionnel d'un atlas linguistique à proprement parler de la même région ${ }^{36}$.

34 Par naturaliste (et naturalisme) nous entendons «plus proche de la réalité directement observable ».

35 En italien il existe un certain nombre de dénominations usuelles (comme p. ex. tesoro, sportello, archivio, etc.), lesquelles, affublées du qualificatif diatopico, pourraient très bien être utilisées dans ce contexte.

36 C'est pourquoi je me trouve en complet désaccord avec la teneur louangeuse d'un article de deux anglicistes (Anderwald/Szmrecsanyi 2008) qui vantent la possibilité de réunir - sous le signe de la linguistique de corpus - un grand nombre d'ethnotextes (en l'occurrence : britanniques) d'origine géographique (et aussi textologique) diverse pour l'analyse "géolinguistique " à proprement parler, et même pour des analyses dialectométriques : cf. Szmrecsanyi 2013. 
Quant à la collecte de données linguistiques structurées - et partant capables de servir de base de données ou de corpus -, il y a - depuis quelque 20 à 30 ans - dans la tête des linguistes, la conviction, complètement erronée, qu'il y en a deux espèces : la soi-disant «bonne» qui consiste à tendre le micro à qui que soit tout en l'invitant à s'exprimer entièrement à son gré, et la « mauvaise » qui prévoit, durant l'enquête, l'observation directe et indirecte de principes et contraintes empiriques préalablement définies $^{37}$.

L'engouement général pour la «bonne» des deux méthodes et le rejet actuel de la "mauvaise » vont à l'encontre de tous les principes de l'observation scientifique tant dans les sciences naturelles que dans les sciences humaines. Toute observation revient à une distanciation de l'objet observé et aussi à un réajustement de ce dernier dans un univers théorique préalablement défini. Or, distanciation et réajustement ne sont ni authentiques ni naturalistes. Toujours est-il que les expériences du bon rendement et de la fécondité de la démarche "contraignante », faites dans beaucoup de sciences au cours de quelque 2500 ans de pratique scientifique, montrent qu'avec une approche moins romantique et plus réfléchie l'on progresse plus vite et avance plus loin.

\section{L'atlas basilectal à l'ère des corpus et des bases de données : l'exemple de l'ALD}

En ce qui concerne notre atlas ALD (résolution italienne du sigle ALD : "Atlante linguistico del ladino dolomitico e dei dialetti limitrofi »), il a été réalisé entre 1985 et 2012 en deux tranches comme le montre le tableau suivant.

Inutile de dire que sa conception est entièrement gilliéronienne. Les principes en sont donc éminemment "géométriques » ou carrément "glotto-géodétiques » Notre propos central n'était donc pas d'augmenter nos connaissances «positives » des dialectes en question ou de dénicher des mots et tournures tout particulièrement « intéressants », mais de rassembler

37 Je précise que parmi les facteurs « contraignants » il se trouve également le fameux « paradoxe de l'observateur ». 


\section{H. GOEBL}

des informations dialectales suffisamment fiables pour permettre l'étude serrée des multiples enchevêtrements et relations qui existent entre les locolectes de l'espace en question. En bref, il s'agissait de préparer tout pour en savoir plus sur la «gestion basilectale de l'espace-ALD » par ses habitants.

Descriptif des deux parties de l'atlas linguistique ALD

\begin{tabular}{|c|c|c|c|}
\hline & Propriété & ALD-I & ALD-II \\
\hline 1 & $\begin{array}{l}\text { Contenu du } \\
\text { questionnaire }\end{array}$ & $\begin{array}{l}\text { Phonétique et } \\
\text { morphologie } \\
\text { (nominale et verbale) } \\
\text { élémentaire }\end{array}$ & $\begin{array}{l}\text { Morphologie } \\
\text { (nominale et verbale) } \\
\text { élaborée, syntaxe et } \\
\text { lexique }\end{array}$ \\
\hline 2 & $\begin{array}{l}\text { Nombre des items } \\
\text { linguistiques du } \\
\text { questionnaire }\end{array}$ & 806 & 1063 \\
\hline 3 & $\begin{array}{l}\text { Items non } \\
\text { linguistiques dans le } \\
\text { questionnaire }\end{array}$ & $\begin{array}{l}\text { Oui : de nature } \\
\text { géographique, } \\
\text { administrative, } \\
\text { ethnographique et } \\
\text { historiques }\end{array}$ & $\begin{array}{l}\text { Oui : de nature } \\
\text { géographique, } \\
\text { administrative, } \\
\text { ethnographique et } \\
\text { historiques }\end{array}$ \\
\hline 4 & $\begin{array}{l}\text { Registre linguistique } \\
\text { visé }\end{array}$ & basilecte & basilecte \\
\hline 5 & Mode d'enquête & $\begin{array}{l}\text { Interviews } \\
\text { standardisées sur le } \\
\text { terrain conduites par } \\
\text { des linguistes }\end{array}$ & $\begin{array}{l}\text { Interviews } \\
\text { standardisées sur le } \\
\text { terrain conduites par } \\
\text { des linguistes }\end{array}$ \\
\hline 6 & $\begin{array}{l}\text { Mode d'élicitation des } \\
\text { réponses }\end{array}$ & $\begin{array}{l}\text { Invitation à ne } \\
\text { produire que des } \\
\text { énoncés jugés } \\
\text { « dialectaux » }\end{array}$ & $\begin{array}{l}\text { Invitation à ne } \\
\text { produire que des } \\
\text { énoncés jugés } \\
\text { « dialectaux » }\end{array}$ \\
\hline 7 & $\begin{array}{l}\text { Nombre minimal de } \\
\text { sujets interviewés par } \\
\text { localité }\end{array}$ & $\begin{array}{l}2 \\
\text { (sociologiquement } \\
\text { différenciés) }\end{array}$ & 1 \\
\hline 8 & $\begin{array}{l}\text { Nombre total des } \\
\text { sujets interviewés }\end{array}$ & 488 & 833 \\
\hline 9 & $\begin{array}{l}\text { Système de } \\
\text { transcription }\end{array}$ & AIS (Ascoli-Böhmer) & AIS (Ascoli-Böhmer) \\
\hline
\end{tabular}




\begin{tabular}{|c|c|c|c|}
\hline 10 & $\begin{array}{l}\text { Données collectées } \\
\text { sur le terrain }\end{array}$ & $\begin{array}{l}\text { Transcriptions, } \\
\text { enregistrements des } \\
\text { interviews, } \\
\text { documentation } \\
\text { ethnophotographique }\end{array}$ & $\begin{array}{l}\text { Transcriptions, } \\
\text { enregistrements des } \\
\text { interviews, } \\
\text { documentation } \\
\text { ethnophotographique }\end{array}$ \\
\hline 11 & $\begin{array}{l}\text { Durée totale des } 217 \\
\text { relevés effectués sur } \\
\text { le terrain }\end{array}$ & $1985-1992$ & $2001-2007$ \\
\hline 12 & $\begin{array}{l}\text { Rapports de travail } \\
\text { périodiques }\end{array}$ & $\begin{array}{l}\text { Oui (dans la revue } \\
\text { «Ladinia ») }\end{array}$ & $\begin{array}{l}\text { Oui (dans la revue } \\
\text { «Ladinia ») }\end{array}$ \\
\hline 13 & $\begin{array}{l}\text { Dates de la } \\
\text { publication de } \\
\text { l'ouvrage imprimé }\end{array}$ & 1998 & 2012 \\
\hline 14 & $\begin{array}{l}\text { Composition de } \\
\text { l'ouvrage imprimé }\end{array}$ & $\begin{array}{l}4 \text { volumes in-folio, } \\
\text { trois volumes avec } \\
\text { des index }\end{array}$ & $\begin{array}{l}5 \text { volumes in-folio, } \\
\text { trois volumes avec } \\
\text { des index }\end{array}$ \\
\hline 15 & $\begin{array}{l}\text { Nombre des planches } \\
\text { linguistiques }\end{array}$ & 884 & 1066 \\
\hline 16 & Outils informatiques & $\begin{array}{l}\text { "Atlas sonore » (sur } \\
\left.\text { DVD et en ligne }{ }^{38}\right) \text {, } \\
\text { base de données } \\
\text { sonore }(\mathrm{SDB}), \text { moteur } \\
\text { de recherche et triage } \\
(\text { IRS })\end{array}$ & $\begin{array}{l}\text { Base de données } \\
\text { sonore }^{39}(\mathrm{SDB}), \\
\text { moteur de recherche } \\
\text { et triage (IRS) }{ }^{40}\end{array}$ \\
\hline 17 & $\begin{array}{l}\text { Site web } \\
\text { multifonctionnel }\end{array}$ & http://ald1.sbg.ac.at/ & http://ald2.sbg.ac.at/ \\
\hline 18 & $\begin{array}{l}\text { Accès ouvert } \\
\text { (public) à la } \\
\text { documentation } \\
\text { imprimée }\end{array}$ & $\begin{array}{l}\text { Non (accès } \\
\text { seulement sur } \\
\text { demande) }\end{array}$ & Oui \\
\hline
\end{tabular}

38 Lien : http://ald.sbg.ac.at/ald/ald-i/.

39 Les deux bases de données sonores (SDB $<$ all. « Sound-Datenbank ») permettent l'accès acoustique direct aux interviews menées par l'insertion du numéro de la localité en question (entre 1 et 217) et de l'item respectif du questionnaire (entre 1 et 1063).

40 Les deux moteurs de recherche et de triage (IRS < angl. « Index Retrieval System ») permettent tant le triage alphabétique (A-Z et Z-A) traditionnel de toutes les formes dialectales figurant sur les planches d'atlas, que le repérage, dans toutes les transcriptions des deux volets de l'ALD, de nexus à librement définir, et ceci dans trois positions différentes à l'intérieur du mot : début, milieu, fin. A l'heure actuelle, les deux logicielsIRS sont très efficaces et rapides. 


\section{H. GOEBL}

Précisons que le réseau d'enquête comprend 217 points d'atlas qui embrassent une superficie de quelque $25000 \mathrm{~km}^{2}$ qui, elle, couvre les régions et cantons italiens et suisses suivants : Lombardie orientale, Grisons orientaux, Trentin, Ladinie dolomitique, Vénétie centrale et septentrionale ainsi que le Frioul occidental. Les enquêtes ne visaient que les prestations basilectales des indigènes telles qu'elles ont été définies par eux-mêmes.

Une autre propriété d'un corpus - dont on parle d'ailleurs peu - est le fait qu'il doit être utilisé (et réutilisé) plus d'une fois, non seulement par son créateur, mais aussi par d'autres chercheurs, aujourd'hui et surtout dans l'avenir. La deuxième de ces deux possibilités suppose une préparation adéquate des structures du corpus en question évidemment en recourant à toutes les possibilités de l'informatique moderne. Sur ce plan, l'atelier de l'ALD a fourni un certain nombre de solutions informatisées dont nous espérons qu'elles pourront servir pour un laps de temps pas trop circonscrit.

Evidemment, quand on prépare un corpus il faut songer aux besoins d'éventuels utilisateurs ce qui, en dernière analyse, est un travail de «théorisation » anticipée. C'est ainsi que pour l'ALD l'utilisateur idéal qui nous a servi de modèle, est non seulement un dialectologue-linguiste animé d'intérêts traditionnels - visant donc à la consultation, ponctuelle et globale, mais toujours limitée, de la documentation visuelle et acoustique de l'atlas - mais aussi quelqu'un qui soit prêt à entreprendre des recherches plus avancées et à se servir directement des deux bases de données (contenant les transcriptions) elles-mêmes ${ }^{41}$.

Malheureusement, devant la caducité notoire de tous les gadgets de l'informatique il est vain de vouloir spéculer sur un avenir lointain. A long terme, la fonctionnalité «tous azimuts » des bases de données sonores (SDB) et des moteurs de recherche (IRS) des deux volets de l'ALD dépendra avant tout du bon fonctionnement des serveurs de l'Université de Salzbourg et de la persistance de la compatibilité - aussi générale que possible - de certaines composantes des logiciels respectifs.

41 Un tel travail a été réalisé par Roland Bauer dans le cadre de la dialectométrisation des données de l'ALD-I (Bauer 2009). 
Toujours est-il que, dans 50 ou 100 ans, des deux parties de l'ALD il ne subsistera que les deux éditions d'atlas en papier, reliées en bleu marin (ALD-I) et en rouge Bordeaux (ALD-II).

Espérons qu'alors leur caractère initial de « corpus » sera toujours évident.

\section{Réflexions finales}

Revenons encore une fois sur la trame métaphorique du réveil de la Belle au bois dormant provoqué par l'intercession d'un prince épris de sa beauté. Les deux pivots substantiels de ce conte sont, d'un côté, l'existence d'un secret qui demande à être découvert, et, de l'autre, celle d'un explorateur qui est poussé par sa curiosité, donc par quelque chose d'inné.

Le prince n'est pas quelqu'un qui flâne d'une façon irréfléchie dans une grande forêt dont il n'a aucune idée, mais, au contraire quelqu'un qui, de la forêt où il s'aventure, a déjà une « certaine idée ». Il en a une théorie.

La Belle au dormant est la cible de cette théorie; ce n'est qu'au moment de sa découverte qu'elle peut révéler toute son essence au prince qui en sera ravi ou déçu, selon le cas. Elle assumera le rôle de la justification d'une vision ou théorie.

Ni Charles Perrault ni les frères Grimm n'en parlent: mais je parie que le prince se servait, dans sa recherche du château de la princesse endormie, d'une bonne carte, d'un compas et d'une paire de jumelles, et qu'il avait raconté, après, son exploit à ses amis pour que d'autres traversées de forêts obscures puissent être faites avec moins de risques.

C'est là le secret de la linguistique de corpus, appliquée à la géographie linguistique.

\section{Références bibliographiques}

AIS = Jaberg K. \& Jud J. (1928-1940). Sprach- und Sachatlas Italiens und der Südschweiz. 8 vol. Zofingen: Ringier (réimpression : Nendeln : Kraus, 1971).

ALD-I = Goebl H., Bauer R., Haimerl E. et al. (éd.) (1998). Atlant linguistich dl ladin dolomitich $y$ di dialec vejins, $1^{a}$ pert/Atlante linguistico del ladino dolomitico e dei dialetti 


\section{H. GOEBL}

limitrofi, $1^{a}$ parte/Sprachatlas des Dolomitenladinischen und angrenzender Dialekte, 1. Teil. 4 vol. in-folio (avec cartes), 3 vol. d'index, 3 CD-ROM. Wiesbaden : L. Reichert.

ALD-II = Goebl H. et al. (éd.) (2012). Atlant linguistich dl ladin dolomitich y di dialec vejins, $2^{a}$ pert/Atlante linguistico del ladino dolomitico e dei dialetti limitrofi, $2^{a}$ partel Sprachatlas des Dolomitenladinischen und angrenzender Dialekte, 2. Teil. 5 vol. in-folio (avec cartes), 3 vol. d'index. Strasbourg : Editions de Linguistique et de Philologie.

ALF $=$ Gilliéron J. \& Edmont E. (éd.) (1902-1910). Atlas linguistique de la France. 10 vol. Paris : Champion (réimpression : Bologna, Forni, 1968).

$\mathrm{ALG}=$ Séguy J. (1954-1974). Atlas linguistique et ethnographique de la Gascogne. 6 vol. Paris : CNRS.

ALPI = Atlas lingüistico de la península ibérica (1962). Vol. I. Fonética. Madrid : CSIC.

ALR = Puşcariu S., Pop S. \& Petrovici E. (éd.) (1938-1942). Atlasul lingvistic român. 2 vol. Sibiu: Muzeul Limbii Române, Leipzig : Harrassowitz.

Anderwald L. \& Szmrecsanyi B. (2008). "Corpus linguistics and dialectology », in Lüdeling A. \& Kytö M. (éd.) Corpus Linguistics. Berlin : Mouton de Gruyter, Handbücher der Sprach- und Kommunikationswissenschaft 29, vol. 2, 11261140.

Ascoli G.I. (1874). « Schizzi franco-provenzali », Archivio glottologico italiano $3: 61-120$.

Bauer R. (2009). Dialektometrische Einsichten. Sprachklassifikatorische Oberflächenmuter und Tiefenstrukturen im lombardo-venedischen Dialektraum und in der Rätoromania. San Martin de Tor: Istitut ladin Micurà de Rü (Ladinia monographica 01).

Billy P.-H. (1993). Index onomasiologique des Atlas linguistiques par régions (domaine gallo-roman), de l'Atlas linguistique de la France et du Französisches etymologisches Wörterbuch, XXI-XXIII. Toulouse : Presses Universitaires du Mirail. 
Brun-Trigaud G., Le Berre, Y. \& Le Dû J. (2005). Lectures de l'Atlas linguistique de la France de Gilliéron et Edmont. Du temps dans l'espace. Essai d'interprétation des cartes de l'Atlas linguistique de la France de Jules Gilliéron et Edmond Edmont augmenté de quelques cartes de l'Atlas linguistique de la Basse-Bretagne de Pierre Le Roux. Paris : CTHS.

Coquebert de Montbret B.-E. \& Bouderie J. (1831). Mélanges sur les langues, dialectes et patois renfermant, entre autres, une collection de versions de la Parabole de l'Enfant Prodigue en cent idiomes ou patois différents, presque tous de France, précédés d'un essai de travail sur la géographie de la langue française. Paris : Delaunay.

Ettmayer K. von (1924). « Über das Wesen der Dialektbildung, erläutert an den Dialekten Frankreichs », Denkschriften der Akademie der Wissenschaften in Wien, philosophischhistorische Klasse, vol. 66/3, 1-56, 7 tableaux.

FEW = Wartburg W. von (1922 ss.). Französisches etymologisches Wörterbuch. Bonn, Berlin, Basel : Klopp, Teubner, Zbinden.

Gilliéron J (1881). Petit atlas phonétique du Valais roman (Sud du Rhône). Paris : Champion (réimpression : Sion : Editions VP, 1997).

Gilliéron J. (1902). Notice servant à l'intelligence des cartes. Paris : Champion. (réimpression : Bologna, Forni, 1968).

Goebl H. (1984). Dialektometrische Studien. Anhand italoromanischer, rätoromanischer und galloromanischer Sprachmaterialien aus AIS und ALF. 3 vol. Tübingen : Niemeyer.

Goebl H. (2002). "Analyse dialectométrique des structures de profondeur de l'ALF », Revue de linguistique romane 66 : $5-63$.

Goebl H. (2003). «Regards dialectométriques sur les données de l'Atlas linguistique de la France (ALF) : relations quantitatives et structures de profondeur », Estudis Romànics $25: 59-96$. 


\section{H. GOEBL}

Goebl H. (2004). « Joseph Durand (de Gros), 1826-1900. Ein weitgehend unbekannter Protagonist in der Ascoli-MeyerDebatte über die Existenz von Dialekten », in A. Gil, D. Osthus \& Cl. Polzin-Haumann (éd.). Romanische Sprachwissenschaft. Zeugnisse für Vielfalt und Profil eines Faches. Festschrift für Christian Schmitt zum 60. Geburtstag. Frankfurt/Main, Berlin : Lang, vol. 1, 169-192.

Goebl H. (2006). "Warum die Dialektometrie nur in einem romani(st)ischen Forschungskontext entstehen konnte », in W. Dahmen, G. Holtus, J. Kramer J., M. Metzeltin, W. Schweickard \& Winkelmann O. (éd.) Was kann eine vergleichende romanische Sprachwissenschaft heute (noch) leisten? Romanistisches Kolloquium vol. XX. Tübingen : Narr, 291-317.

Goebl H. (2008). « La dialettometrizzazione integrale dell'AIS. Presentazione dei primi risultati », Revue de linguistique romane $72: 25-113$.

Goebl H. (2010). «La concezione ascoliana del ladino e del franco-provenzale ", in Marcato C. / Vicario F. (éd.). Il pensiero di Graziadio Isaia Ascoli a cent'anni della scomparsa. Convegno internazionale, Gorizia-Udine, 3-5 maggio 2007. Udine : Società Filologica Friulana, 147-175.

Jaberg K. (1906). "Zum Atlas linguistique de la France », Zeitschrift für romanische Philologie $30: 512$.

Jaberg K. (1908). Sprachgeographie. Beitrag zum Veständnis des Atlas linguistique de la France. Aarau: Sauerländer (traduction espagnole: Geografía lingüística. Ensayo de interpretación del "Atlas lingüístico de Francia». Traducción de A. Llorente y M. Alvar. Granada : Universidad de Granada, 1959).

Jaberg K. (1903). Aspects géographiques du langage. Paris : Droz.

Jaberg K. \& Jud J. (1928). Der Sprachatlas als Forschungsinstrument. Kritische Grundlegung und Einführung in den Sprach- und Sachatlas Italiens und der Südschweiz. Halle : Niemeyer (réimpression : Nendeln : Kraus, 1973; traduction italienne : L'atlante linguistico come strumento di ricerca. 
Fondamenti critici e introduzione. Edizione italiana a cura di G. Sanga e S. Baggio. Milano : Unicopli, 1987).

Lauwers P., Simoni-Aurembou M.-R. \& Swiggers P. (éd.) (2002). Géographie linguistique et biologie du langage. Autour de Jules Gilliéron. Leuven/Louvain. Paris, Dudley, M.A : Peeters.

Lequin G. (1924). Georges Protat (1857-1923). Mâcon : Protat frères.

Meyer P. (1875). Compte rendu de: Ascoli 1874, Romania 4 : 293-296.

NADIR = Sobrero A., Romanello M. T. \& Tempesta I. (1991). Lavorando al NADIR. Un'idea per un atlante linguistico. Galatina : Congedo.

Ozouf-Marignier M.-V. (1989). La formation des départements : représentions du territoire français à la fin du XVIII siècle. Paris : Editions de l'Ecole des Hautes Etudes en Sciences Sociales.

Palsky G. (1996). Des chiffres et des cartes. Naissance et développement de la cartographie quantitative française au $X I X^{e}$ siècle. Paris : CTHS.

Pop S. (1950). La dialectologie. Aperçu historique et méthodes d'enquêtes linguistiques. 2 vol. Louvain: chez l'auteur, Gembloux : Duculot.

Pop S. \& Pop R. D. (1959). Jules Gilliéron. Vie enseignement, élèves, oeuvres, souvenirs. Préface de Pierre Chantraine. Louvain : Centre International de dialectologie générale.

Ravier X. (1976). "Jean Séguy et la traversée du langage gascon. Réflexions sur une topogenèse géolinguistique », Revue de linguistique romane $40: 389-402$.

Robinson A. H. (1982). Early thematic mapping in the history of cartography. Chicago, London: University of Chicago Press.

Roncayolo, M. (1992). «Le département», in Nora P. (éd.). Les lieux de mémoire. Vol. 3, 1 : Les France. Paris : Gallimard, 884-929. 


\section{H. GOEBL}

Séguy J. (1971). "La relation entre la distance spatiale et la distance lexicale », Revue de linguistique romane 35 : 335357.

Séguy J. (1973). «La dialectométrie dans l'Atlas linguistique de la Gascogne », Revue de linguistique romane 37 : 1-24.

Simoni-Aurembou M.-R (1989). « La couverture géolinguistique de l'Empire français : l'enquête de la Parabole de l'Enfant Prodigue », in Espaces romans. Etudes de dialectologie et géolinguistique offertes à Gaston Tuaillon. Grenoble: ELLUG, vol. 2, 114-139.

Storost J. (2008). In memoriam Vladimiro Macchi. Aspekte der Wissenschaftsgeschichte. Ausgewählte Sujets. Bonn : Romanistischer Verlag.

Szmrecsanyi B. (2013) Grammatical Variation in British Dialects. A Study in Corpus-Based Dialectometry. Cambridge. Cambridge University Press.

Tourtoulon Ch. de \& Bringuier O. (1876). «Rapport sur la limite géographique de la langue d'oc et de la langue oïl », Archives des Missions scientifiques et littéraires III/3: 544-605, 1 carte.

Tufte E. R. (1997). Visual Explanations. Images and Quantities, Evidence and Narrative. Cheshire, Connecticut: Graphics Press. 